\title{
OSSERVAZIONI IN OCCASIONE DI UNA VISITA AI CROATI DEL MOLISE (ITALIA)
}

Chi venendo da Vasto, città situata sulla costa adriatica, dopo circa venti chilometri lascia la strada per Isernia in direzione sud, per fare una scappata a San Felice del Molise si accorgerà difficilmente di una qualche particolarità che distingua questo paese di circa 900 abitanti da altre località meridionali.

Può darsi che, quando va a passegio per il paese, in Via Roma attiri la sua attenzione un cartello sulla facciata di una casa e che porta l'iscrizione

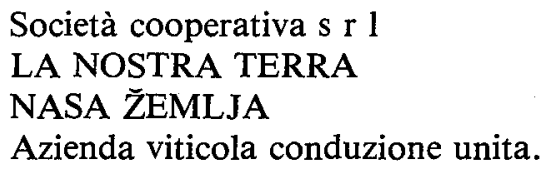

Leggendo la terza riga forse rimane sorpreso: due parole che né sono italiane né possono essere annoverate ai dialetti molisani vicini. Nasa žemlja, la traduzione letterale di la nostra terra, è serbo-croato.

Se si chiede il significato di questa iscrizione slava, gli abitanti spiegano volontieri allo straniero, che San Felice (o Sti Filič, come viene ancora chiamato dagli anziani) è uno di quei paesi, dove nei secoli 15. e 16. dei Croati si stabilirono dopo la fuga davanti ai Turchi.

A Montelungo, Mafalda, S. Biave e Tavenna ${ }^{1}$ già da molto tempo non si parla più croato; diversamente a S. Felice, Acquaviva Collecroce e Montemitro - tuttavia si deve prendere atto del fatto che le popolazioni di queste tre località molisane restano fedeli al dialetto originario in diversa misura.

A Montemitro (nome croato Mundimitar) praticamente tutti i 550 abitanti (popolazione presente, 25 ottobre 1985$)^{2}$ parlano ancora croato in tutte le situazioni della vita comune, eccezion fatta delle persone immigrate per matrimonio. Per i bambini la prima lingua - quindi la lingua madre - è come sempre, il croato. Nel paese si trovano diversi segnali d'indicazione bilingui ${ }^{3}$.

1 Nel dialetto di Tavenna si trovano ancora sporadici lessemi croati, p. e. bátsa 'fratello maggiore', šóša 'sorella'. Nel 1864 a Tavenna 60 persone anziane parlavano ancora croato, mentre la lingua si era spenta a S. Biave verso l'anno 1850 e a Mafalda prima del 1780 . A Montelungo già nel 1790 non ci si ricordava più dell'origine slava del paese. Cf. G. Buratti, „Die Kroaten des Molise (Italien)“, in: M. Straka (ed.), Handbuch der europäischen Volksgruppen, Wien/Stuttgart 1970, pp. 493-495.

2 Cf. appendice 2. Secondo un censimento privato del 19544.036 dei 4.504 abitanti delle tre località dovrebbero essere stati ancora slavofoni. Cf. Buratti, op. cit., p. 494.

3 P. e.: cappella s. lucia (kapela sv. luca); municipio (općina); scuola elementare (škola); centro - centar. 
Ad Acquaviva Collecroce 4 (popolazione presente 1981: 984) si può constatare, che la maggior parte della popolazione nella comunicazione quotidiana usa il croato. Potei sincerarmi che durante una partita di calcio della squadra di San Felice contro la squadra di un paese vicino che fu disputata ad Acquaviva, i giovani tifosi non solo chiacchieravano e bestemmiavano ma anche incitavano la squadra di $\mathrm{S}$. Felice in croato (accanto alle bandiere italiane sventolavano delle bandiere jugoslave al vento). Lo stesso vale per la conversazione sulla strada, al bar, durante la partita di carte ecc. Tuttavia si troveranno appena bambini con meno di dieci anni che parlano croato, anche se lo capiscono!

Del tutto diversa la situazione a San Felice. Qui di regola solo la generazione anziana parla ancora croato. Le generazioni giovane e media hanno già adottato il dialetto molisano, anche se per lo più possiedono ancora conoscienze passive (secondo età) del croato. A Montemitro invece il dialetto regionale è praticamente sconosciuto.

Una causa particolare per il fatto che San Felice si è dimostrato il meno resistente contro influssi dialettali forestieri si può trovare nello stabilirsi di circa 150 abitanti di Casoli (circa 30 chilometri al sud di Chieti) alla fine degli anni 20 sul territorio del comune (però alcuni chilometri fuori del centro abitato) - così la popolazione di S. Felice entrò in contatto con il loro dialetto. Recentemente alcuni di questi Casolani hanno aperto dei negozi nel paese.

In contrasto con Acquaviva e Montemitro a S. Felice però il serbo-croato viene insegnato nelle classi 2 a 5 della scuola elementare, in seguito a una iniziativa personale dell'insegnante Angelo Genova.

Nei 120 anni passati tutt'e tre i paesi hanno dovuto registrare delle cifre abbastanza forti di emigranti:

Numero di abitanti ${ }^{5}$

$1863 \quad 1911 \quad 1981$

$\begin{array}{lllr}\text { Acquaviva } & 3.291 & 2.243 & 984 \\ \text { San Felice } & 2.337 & 1.681 & 915 \\ \text { Montemitro } & 1.663 & 1.017 & 550 \\ \text { totale } & 7.291 & 4.941 & 2.449\end{array}$

4 Il paese viene chiamato in croato Zivavoda Brdodokriz o brevemente Kruč. Sulla facciata di una casa nel centro del paese si trova un cartello (però non ufficiale), lungo circa $4 \mathrm{~m}$. e alto circa $60 \mathrm{~cm}$, che accanto al nome italiano del paese mostra l'iscrizione DOBRO DOSLI U KRUČ (benvenuti a Kruč). L'unico indizio scritto per il croato è la scrittura MESNIZ (MEŠNIZ sarebbe corretto) sopra l'insegna luminosa di una MACELLERIA.

5 Cifre degli anni 1863 e 1911 secondo G. Masciotta, Il Molise dalle origini ai nostri giorni, vol. I: La provincia di Molise, ristampa: Campobasso 1981 (Napoli 1915), pp. 178 e 181; per l'anno $1981 \mathrm{cf}$. appendice 2 . 
La problematica della disoccupazione nel Mezzogiorno è conosciuta ed è stata descritta sufficientemente.

Chi oggidì visita questi tre paesi durante i mesi d'estate, trova bastanti macchine con targhe tedesche, belghe, svizzere per comprendere le connessioni ${ }^{6}$.

Dopo che nel 1971 a Termoli fu aperto uno stabilimento della FIAT, molti uomini hanno trovato un posto di lavoro in questa fabbrica, cosa che rallentò per il momento il ritmo di emigrazione.

A lungo termine però questi preferiscono un trasloco (a Termoli dove la ditta ha fatto impiantare delle case prefabbricate per $i$ lavoratori) al tragitto quotidiano (distanza Acquaviva - Termoli $40 \mathrm{~km}$, San Felice - Termoli circa $50 \mathrm{~km}$, con in parte cattive condizioni stradali). Da Acquaviva circa 20 impegiati della FIAT con le loro famiglie sono emigranti!

Siccome per lo più delle famiglie giovani emigrano, il quoziente di natalità nei tre paesi croati è bassissimo. Montemitro ha potuto registrare solo quattro nascite nei primi otto mesi del 1985. Questa bassa natalità si rispecchia anche nel numero degli alunni. Nel 1985 solo dieci bambini hanno frequentato la scuola materna di Montemitro (nel 1981 erano 14); per l'anno scolastico 1985/86 cinque scolari si sono icritti per la prima classe della scuola elementare. Il numero di iscrizioni alla prima classe da aspettarsi per l'anno scolastico 1986/87 era già conosciuto nel 1985: sono due. Nelle 5 classi della scuola elementare in tutto venti scolari sono istruiti (di volta in volta due classi sono riunite).

Ad Acquaviva sono state registrate quattro iscrizioni per la prima elementare, a San Felice il numero è relativamente alto, cioè 12 .

Anche la struttura di età in questo contesto lascia riconoscere una situazione piuttosto precaria (riferito al 1981; cf. tavola $4 \mathrm{~A}$ dell'appendice 2).

Ad Acquaviva il numero delle persone appartenenti a una classe d'età (sempre cinque annate unite) è senza eccezione sopra 60 (non contate le persone dai 35 ai 39 anni e quelle dai 40 ai 44 anni, il cui numero è ridotto a causa della guerra). Solo il gruppo dei bambini con meno di 5 anni $(0-4$ anni: 48) e dai 5 ai 9 anni (53) è minore.

A San Felice la tendenza a una denatalità sembra essere incominciata molto prima. A prescindere dalla classe dai 5 ai 9 anni (59!), tutte le classi di età con meno di

\footnotetext{
6 Molte persone sono emigrate oltremare (soprattutto Australia); normalmente non tornano più (al massimo una o due volte per passare le vacanze nel luogo natio). Ma anche i rimpatriati da altri paesi europei spesso preferiscono stabilirsi in località che dispongono di infrastrutture migliori, come possibilità d'istruzione per i bambini (p. e. Termoli).
} 
25 anni hanno meno di 50 membri, la classe delle persone dai 25 ai 29 anni ne ha esattamente 50 .

Tenendo conto del fatto che gli anni della guerra hanno mostrato una denatalità abbastanza forte e che i gruppi con più di 55 anni sono stati decimati a causa della guerra, si deve constatare che le classi sopra menzionate sono inferiori alla media.

Valutando la situazione in un modo alquanto realistico si può osservare che in tutt'e tre i paesi complessivamente $\mathbf{2 . 0 0 0}$ persone parlano ancora croato nelle normali situazioni di comunicazione. Appena è presente un forestiero, il gruppo parla italiano o dialetto regionale. Tra persone croatofone di provenienza diversa, già oggi non è più sicuro che la comunicazione si sviluppi in croato.

Quali prognosi si possono fare per il futuro? Con qualche probabilità si può dire che a Montemitro e forse anche ad Acquaviva si parlerà croato fino al 21. secolo avanzato. Per quanto riguarda San Felice le prospettive sono piuttosto tristi; con la generazione anziana muore anche il croato, tanto più che i giovani sollevano sempre la questione dell',utilità “7.

Finché i Croati del Molise non sono riconosciuti dallo Stato italiano cone minoranza etnica e linguistica ${ }^{8}$, non si avrà un insegnamento regolare della lingua e nella lingua ${ }^{9}$.

A San Felice la lingua non mi sembra possa più essere salvata. Per Acquaviva le prospettive sono ancora buone, ma il tempo stringe. Montemitro non è - almeno finora - minacciato. E' vero che una proposta di legge è stata presentata al parlamento, ma è dubbio se verrà votata (cf. appendice 1).

Momentaneamente esiste soltanto la possibilità per 30 giovani di partecipare a corsi di lingua a Zagabria (le spese insorgenti vengono assunte dalla Jugoslavia). Nel 1983 solo 12 giovani sfruttarono l'occasione, 30 nel 1984 e 23 nel 1985 (tuttavia rimane dubbio, se l'apprendimento del serbo-croato moderno possa contribuire al

7 P. Piccoli di Acquaviva (intervista del 1. 9. 1985): „Uno è interessato in proporzione a quanto la lingua può essere utile per il lavoro, per i rapporti con gli altri. Per il lavoro la lingua attualmente non serve, non dà nessuna possibilità di lavoro in più. Nei rapporti sociali parliamo sempre in croato, sempre che non sia necessario sostituire l'italiano per mancanza di termini. Con gente di Montemitro si parla in croato."

8 „Non venendo riconosciuta come minoranza, non creandosi delle prospettive economiche, attualmente si tende a emigrare, sia da parte di coloro che studiano che sia da parte dei figli di agricoltori ... Se queste nuove generazioni vanno via, è normale che la lingua avrà meno possibilità di sviluppare." (P. Piccoli, 1. 9. 1985).

9 I nuovi programmi della scuola elementare, approvati nel 1985 prevedono l'insegnamento di una seconda lingua nella scuola elementare. Questa potrebbe essere I'occasione per insegnare il croato, se i genitori lo chiedono; però è quasi sicuro che i genitori chiederanno l'inglese o il francese come lingue più a diffusione internazionale. Un altro problema sarebbe la mancanza di persone capaci di insegnare la madrelingua, la mancanza di una grafia ecc. Inoltre manca un vocabolario, mancano termini astratti, tecnici ecc. 
mantenimento della lingua madre). Oltre questa ci sono poche iniziative per la conservazione della lingua. Nel marzo del 1985 il „Periodico dei Paesi del Molise“ (Glasnik molizanskih hrvata) NAS̆ JESIK (la nostra lingua) fu pubblicato per prima volta dopo una pausa di molti anni (la rivista era uscita dal 1967-1970). Fu curato dall'Associazione culturale „Naš Grad“ (Via S. Angelo, N. 74, I-86030 Acquaviva Collecrocce, Provincia di Campobasso). Speriamo che NAS̆ JESIK ridiventi una impresa fissa e alla memoria della popolazione di Sti Filič, Mundimitar e Kruč si vorrebbero richiamare le parole di N. Neri (1761-1799) di Acquaviva, morto nella Rivoluzione Napoletana: „Nomo ta zabit naš lipi jesik! “ — „Non dimenticate la nostra bella lingua!“ (NAS̆ JESIK, marzo 1985, p. 1) ${ }^{10}$.

\section{Appendice 1}

Disegno di legge „tutela delle minoranze linguistiche“, testo approvato il 17 aprile 1985 dalla Commissione Affari Costituzionali della Camera (in tutto 19 articoli; abbreviato considerevolmente)

Art. 1

La Repubblica tutela la lingua e la cultura delle popolazioni di origine albanese, catalana, germanica, greca, slava e zingara e di quelle parlanti il ladino, il francoprovenzale e l'occitano. sarde.

La Repubblica tutela, altresì, la lingua e la cultura delle popolazioni friulane e

Art.3

Nelle scuole materne ed elementari dei comuni indicati nel decreto del Presidente della Giunta regionale . . ., l'educazione linguistica prevede l'apprendimento della lingua locale e l'uso della stessa, in via strumentale, al fine della migliore cognizione delle materie, nonché l'insegnamento delle forme espressive dell'infanzia, la lettura e le esercitazioni relative agli argomenti concernenti gli usi, i costumi e le tradizioni delle comunità locali.

Nelle scuole medie dell'obbligo degli stessi comuni è previsto l'insegnamento della lingua locale a richiesta degli interessati.

I programmi e gli orari relativi all'educazione linguistica saranno fissati con decreto del Ministro della Pubblica Istruzione, sentito il Consiglio nazionale della Pubblica Istruzione e tenuto conto dei criteri di gradualità in relazione alla disponibilità di personale insegnante e di materiale didattico.

10 Un altro "numero unico in attesa di autorizzazione“ di NAŠ JEZIK (questa volta con $z$ ) usci nell'ottobre 1985. 
Art. 4

Nelle scuole elementari e medie dei comuni indicati nel decreto del Presidente della Giunta regionale ... ., la cultura e le tradizioni locali costituiscono materia di insegnamento obbligatorio nell'ambito degli insegnamenti di storia, geografia, educazione musicale, artistica e tecnica.

La disposizione di cui al primo comma si applica ai corsi dello stesso livello svolti per i lavoratori presso le scuole statali nonché ai corsi di educazione permanente.

Art. 7

. . . i membri dei consigli comunali e circoscrizionali e degli organi circiscrizionali della scuola possono usare la lingua locale nell'attività degli organi medesimi.

Quando non sia possibile disporre di un servizio di traduzione, sono prive di effeti giuridici le dichiarazioni che non siano espresse anche in lingua italiana.

Art. 9

Nei comuni indicati nel decreto del Presidente della Giunta regionale . . ., al fine di agevolare il rapporto dei cittadini, è consentito l'uso orale della lingua ammessa a tutela negli uffici d'amministrazione pubblica.

Art. 10

Nei comuni indicati nel decreto del Presidente della Giunta regionale . . ., in aggiunta ai toponimi ufficiali, i consigli comunali possono deliberare l'adozione di toponimi conformi alle tradizioni e agli usi locali, secondo modalità stabilite con legge regionale.

Per il testo integrale cf. NAŠ JEZIK, ottobre 1985, pp. 4-5. 
Appendice 2: $12^{\circ}$ censimento della popolazione, 25 ottobre 1981, Volume II: dati sulle caratteristiche strutturali della popolazione e delle abitazioni, Tomo I - Fascicoli provinciali, 70 - Campobasso, Istitutto Centrale di Statistica, Roma 1983.

Tavola 1: Superficie territoriale e densità - Popolazione residente e popolazione presente, per sesso (pp. 2-3)

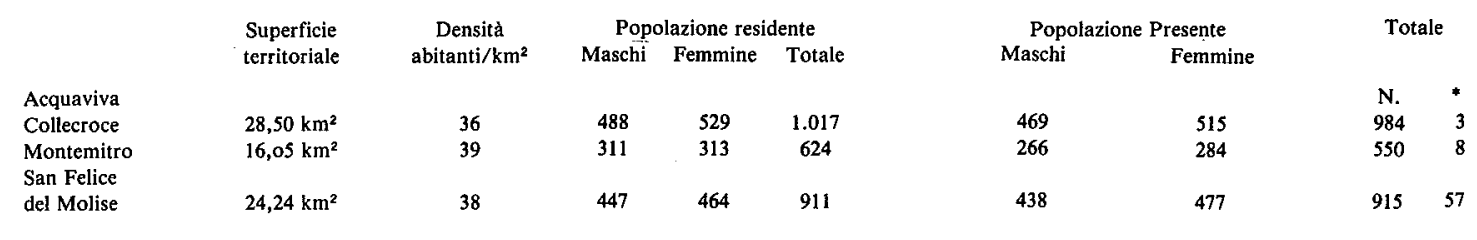

* di cui temporaneamente presente

Tavola 2: Popolazione residente temporaneamente assente per sesso, luogo di presenza e motivo dell'assenza (pp.4-5)

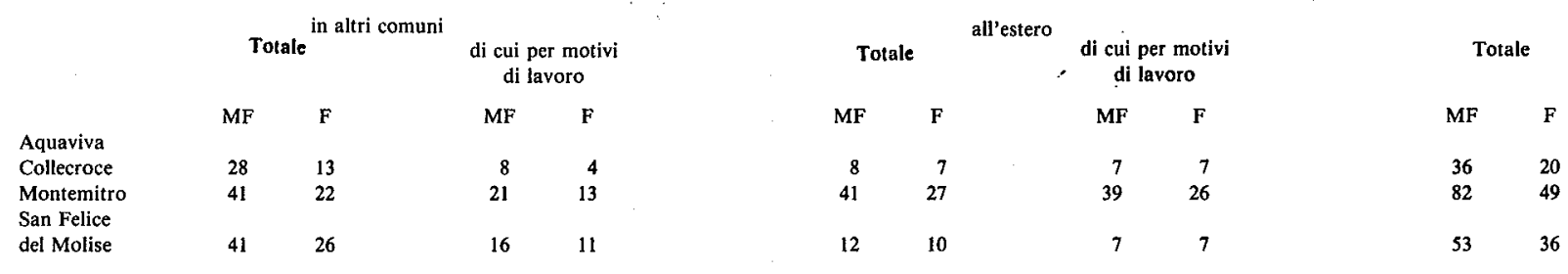

Tavola 4 A: Popolazione residente per sesso e classe di età (pp. 8-9)

\begin{tabular}{|c|c|c|c|c|c|c|c|c|c|c|c|c|c|c|c|c|c|}
\hline & meno di 5 & $5-9$ & $10-14$ & $15-19$ & $20-24$ & $25-29$ & $30-34$ & $35-39$ & $40-44$ & $45-49$ & $50-54$ & $55-59$ & $60-64$ & $65-69$ & $70-74$ & 75 e piu & totale \\
\hline A. & 48 & 53 & 71 & 61 & 70 & 63 & 61 & 28 & 46 & 69 & 64 & 63 & 72 & 79 & 79 & 90 & 1.017 \\
\hline M. & 28 & 36 & 35 & 53 & 43 & 35 & 37 & 15 & 39 & 49 & 54 & 41 & 49 & 41 & 35 & 36 & 624 \\
\hline SF. & 46 & 59 & 44 & 47 & 44 & 50 & 59 & 33 & 47 & 54 & 63 & 73 & 61 & 71 & 74 & 86 & 911 \\
\hline
\end{tabular}


Tavola 7: Popolazione residente attiva e non attiva per sesso (pp.28-31)

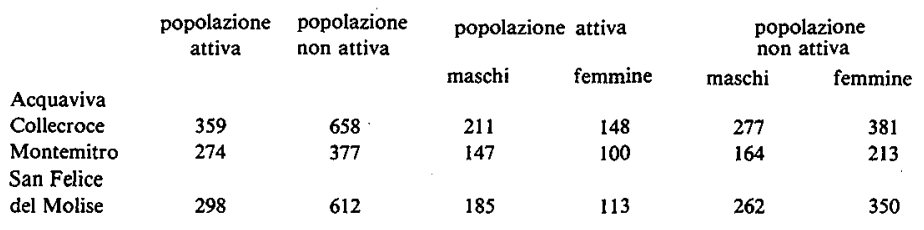

Tavola 15: Abitazioni in complesso (occupate e non occupate) (pp. 76-79)

$\begin{array}{lccc} & \begin{array}{c}\text { abitazioni } \\ \text { occupate }\end{array} & \begin{array}{c}\text { abitazioni } \\ \text { non occupate }\end{array} & \text { totale } \\ \text { Acquaviva } & & 140 & 493 \\ \begin{array}{l}\text { Collecroce } \\ \text { Montemitro }\end{array} & 353 & 94 & 310 \\ \begin{array}{l}\text { San Felice } \\ \text { del Molise }\end{array} & 216 & 150 & 473\end{array}$

\section{Povzetek}

OB OBISKU PRI HRVATIH V POKRAJINI MOLISE (ITALIJA)

Avtor daje statistične podatke, ki zadevajo hrvaško etnijo $v$ treh vaseh južnoitalijanske pokrajine Molise; gre za danes številčno majhen ostanek vala beguncev, ki so se pred turško nevarnostjo v 15 . in 16. stoletju zatekli v Južno Italijo in svoj govor še obdržali. Upošteva statistične podatke zadnjega క̌tetja v Italiji, leta 1981; ceni število tistih, ki v družini še govorijo srbohrvaško, na kakih dva tisoč. 